\title{
Application of Bilateral De-epithelialized Hinge Flap to Correct the Deep Depressed Scar
}

\author{
So-Min Hwang, Sang-Hwan Lee, \\ Hong-Il Kim, Yong-Hui Jung, \\ Hyung-Do Kim
}

Aesthetic, Plastic \& Reconstructive Surgery Center, Good Moonhwa Hospital, Busan, Korea

No potential conflict of interest relevant to this article was reported.

\begin{abstract}
Background Depressed scars are usually corrected using subcutaneous fillers such a dermal fat grafting or an autologous fat grafting. But, fillers are absorbed over time and cannot be appropriately used for the correction of large or deep depressed scars. Therefore, we tried new methods to correct challenging cases of deep depressed scars.

Methods From 2005 to 2013, deep depressed scar were corrected in 10 patients. The location of the scars were as follows: 6 neck, 4 lower extremities. Preoperative marking was done involving the scar on the skin in an oval shape including the long axis of relaxed skin tension line, and the epithelium of the scar was removed. Both ends of the dermal layer of the residual scar were dissected and then isolated from the subcutaneous layer of the adjacent skin. Then, we folded both ends of the flap like hinge flaps and thereby appropriately restored the depressed part of the dermal layer. And we directly closed skin for scar revision.

Results All ten patients had a good result and satisfaction without complication, such as wound infection, dehiscence, at a mean follow-up of seven months. Correction of depression area made the depressed scar less noticeable. Moreover, the problems like donor morbidity, color and texture matching in case of using our techniques, are not issued.

Conclusions We folded residual scar tissue like a hinge flap in the correction of depressed scar. We obtained good outcomes and report our new methods and their outcomes with a review of literatures.
\end{abstract}

Keywords : Cicatrix, Scar, Hinge

\section{INTRODUCTION}

The scar is formed following the trauma or severe inflammation, and it is one of the critical problems in the field of plastic and reconstructive surgery. To date, continuous efforts have been made

Received: Sep 23, 2013 Revised: Oct 16, 2013 Accepted: Oct 18, 2013 Correspondence: So-Min Hwang Aesthetic, Plastic \& Reconstructive Surgery Center, Good Moonhwa Hospital, 119 Beomil-ro, Dong-gu, Busan 601-803, Korea. E-mail: Sangwind@hanmail.net

http://submit.e-aaps.org/

Copyright (๑) 2013 The Korean Society for Aesthetic Plastic Surgery.

This is an Open Access article distributed under the terms of the Creative Commons Attribution Non-Commercial License (http://creativecommons.org/licenses/by-nc/3.0/) which permits unrestricted non-commercial use, distribution, and reproduction in any medium, provided the original work is properly cited to correct scar. Still, however, it remains challenging to remove scar completely. The scar formation is a process that occurs during the wound healing, which is characterized by the synthesis of abnormal collagen and irregular changes on the skin surface [1]. Various factors that may affect the wound healing process, leads to the formation of various types of scars such as linear scar and depressed one. Most cases of the linear scars are corrected using a scar revision. This leads to good treatment outcomes. In addition, small-sized depressed scars are corrected using a dermal-fat grafting or an autologous fat grafting [2]. But the fillers are disadvantageous in that the rate of absorption is relatively higher and additional surgeries are required to provide filling materials. Various methods have therefore been used to correct deep depressed scars. But the correction of deep depressed scars poses challenging problems for plastic surgeons. In the past, de-epithelialized scar 



A


B

Fig. 1. Schematic diagrams. (A) This is such a method that can correct the depressed scars by folding the tissue. It is also disadvantageous, however, in that new donor sites are needed and the skin graft is needed to cover the surface of the donor flaps. (B) The scars may be used as a flap without providing new donor sites in the correction of depressed scar. It can therefore be inferred that it would be possible to correct the depressed scars without providing the donor flaps if surgeons fold the scar tissue like a hinge flap in the correction of deep scar tissue.

tissue has been used to correct the depressed scar because residual scar tissue is helpful for the correction of depression [3,4]. And hinge flap was also used to correct deep depressed scar such as the post-tracheostomy scar by turning over flap like the page of the book and skin graft (Fig. 1A) [5]. By the application of two methods, we find a simple and effective way to correct the deep depressed scar. We used the methods that folded bilateral de-epithelialized residual scar tissue like a hinge flap in the correction of depressed scar (Fig 1B). Therefore, we obtained the good outcomes of the correction of deep depressed scars. So, we introduce new-method to correct deep depressed scar.

\section{METHODS}

The current study was conducted in ten patient with deep depressed scar from 2005 to 2013. The patients included 6 males and 4 females with an average age of 49 years. The location of the scars were as follows: 6 neck, 4 lower extremities (Table 1). The patients wanted to improve the depression. After surgery, all the patients received local wound management and antibiotic administration.

Preoperative marking was done involving the depressed area on the skin in an oval shape. This was done following the relaxed skin tension line, if that's possible. We made a careful incision according to the preoperative marking. The epithelium of scar was removed using a sharp scalpel and a scissor (Fig. 2A). Then, both
Table 1. Summary of patients characteristics

\begin{tabular}{ccccc}
\hline Cases & Sex/Age & Scar location & Scar size $(\mathrm{cm})$ & $\begin{array}{c}\text { Preoperative marking } \\
\text { following RSTL }\end{array}$ \\
\hline 1 & M/44 & Neck & $2.0 \times 2.0$ & 0 \\
2 & $M / 58$ & Neck & $2.5 \times 2.0$ & 0 \\
3 & $M / 57$ & Neck & $2.0 \times 1.5$ & 0 \\
4 & $M / 59$ & Neck & $2.0 \times 2.0$ & 0 \\
5 & F/58 & Neck & $2.0 \times 1.5$ & 0 \\
6 & F/57 & Neck & $1.5 \times 1.0$ & 0 \\
7 & $M / 39$ & Lower extremity & $5.0 \times 2.5$ & $\mathrm{X}$ \\
8 & $\mathrm{M} / 37$ & Lower extremity & $5.0 \times 2.0$ & $\mathrm{X}$ \\
9 & $\mathrm{~F} / 32$ & Lower extremity & $6.0 \times 2.0$ & $\mathrm{X}$ \\
10 & $\mathrm{~F} / 49$ & Lower extremity & $4.5 \times 2.0$ & 0 \\
\hline
\end{tabular}

RSTL, relaxed skin tension line.

ends of the dermal layer of the residual scar were dissected and isolated from the subcutaneous layer of the adjacent tissue. With the elevation of bilateral dissected flaps, accompanied by the preservation of the approximately middle $1 / 3$ of the center in depressed area, attempts were made to prevent the hemodynamic derangement (Fig 2B). Thus, we folded both ends of the flap sharing the central region and thereby appropriately restored the depressed part of the dermal layer (Fig 2C). This was followed by suturing with a 4-0 PDS at the sites where the both ends of bilateral hinge flaps met. If amounts of scar tissue were insufficient for cor- 



Fig. 2. Intra-operative findings. (A) The cicatrical island formed after de-epithelialization of the depressed scar. (B) The central $1 / 3$ of this area should remain undisturbed, if possible, because it constitutes the vascular pedicle of the flap. The surrounding skin edges were undermined, and the end of both part of cicatricial island was dissected and elevated. (C) To correct the depression, the end of both parts of de-epithelialized cicatricial island was turned towards the center of islands like the hinge flap.
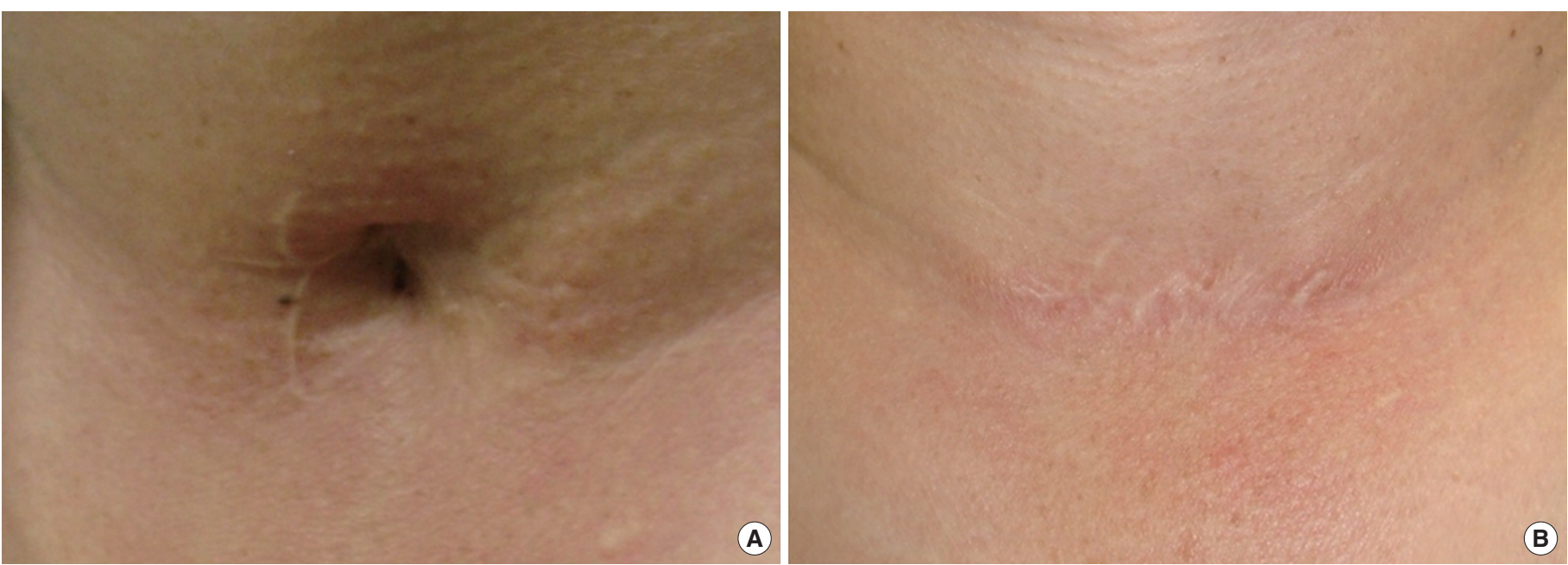

Fig. 3. Preoperative and postoperative clinical photograph. (A) Preoperative photograph showing the depressed scar in the suprasternal notch after tracheostomy. (B) Postoperative photograph at one year and four months showing good wound healing.

recting the center of depression, we overlaped the both ends of bilateral hinge flaps. We approached the skin by undermining the subcutaneous tissue of adjacent normal tissue, and we directly closed skin for scar revision. The sutures were removed on tenth day following surgery.

\section{RESULTS}

All ten patients had a good result and satisfaction without complication, such as wound infection, dehiscence, depression or shrinkage at a mean follow-up of seven months. Correction of depression area made the depressed scar less noticeable. Moreover, the problems like donor morbidity, color and texture matching in case of using our techniques, are not issued.

\section{Case I}

Six years ago, a 44-year-old man had breathing disorder caused by occupational injury, and he had undergone tracheostomy. The patient's respiratory problems were resolved and tracheostomy was removed. But, he visited us with a chief complaint of depressed scar formed on the neck (Fig. 3A). He underwent correction of depressed scar using the bilateral de-epithelialized hinge flap. One year and four months postoperatively, at a follow-up, he achieved a correction of the depressed scar (Fig. 3B). Patients were satisfied with treatment outcomes.

\section{Case II}

A 32-year-old woman visited us with a chief complaint of deep depressed scar formed in the left thigh (Fig. 4A). The patient had un- 

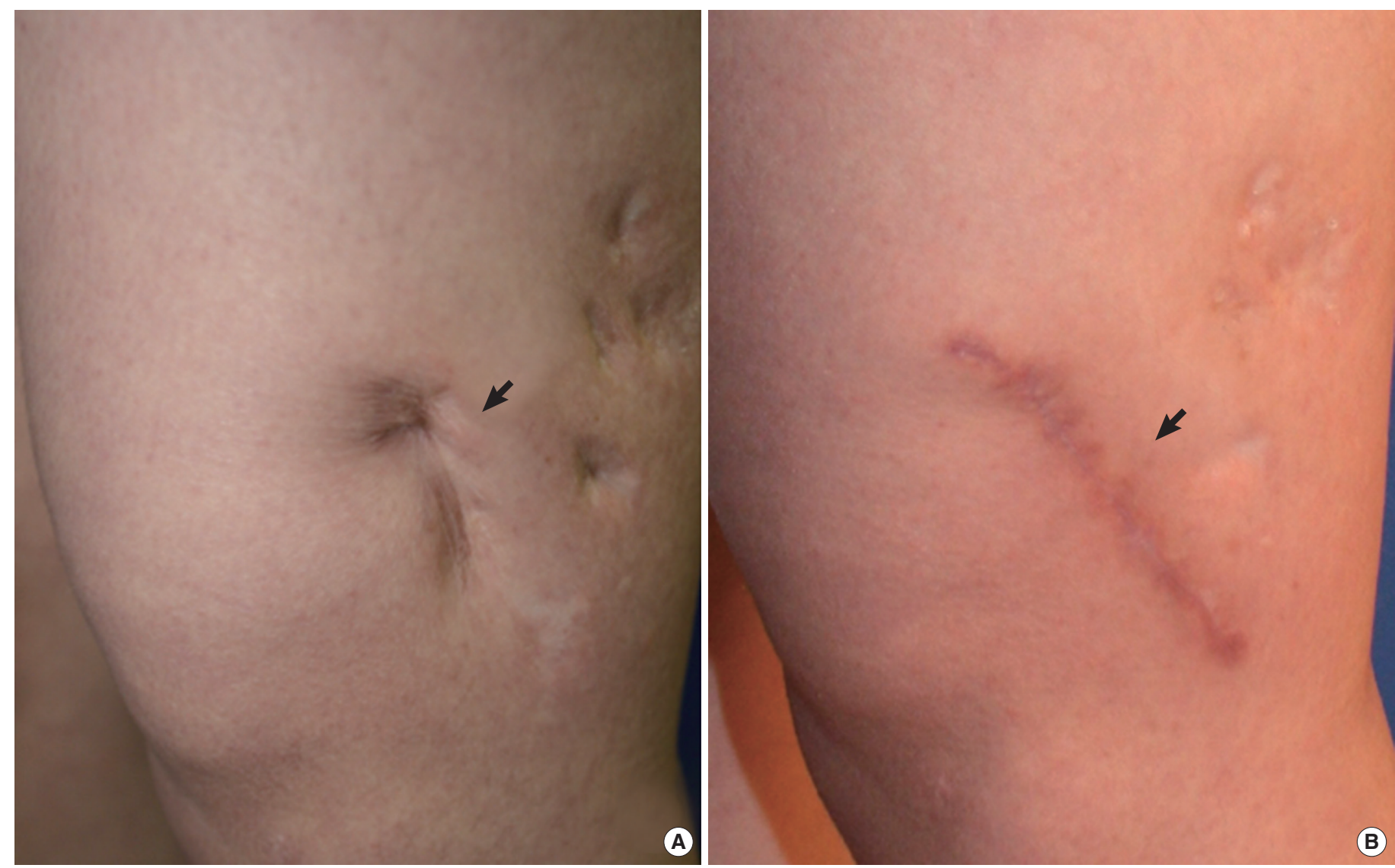

Fig. 4. Preoperative and postoperative clinical photographs. (A) Preoperative presence of a 6-cm deep depressed scar in the left thigh after Ilizarov operation. (B) Postoperative photograph at four months showing good wound healing.

dergone Ilizarov surgery for the correction of the congenital dislocation of the hip five years ago. Two years later, the patient had undergone surgery for the removal of pins and an external fixator. But the severe depressed scar was presented. She underwent correction of depressed scar using the bilateral de-epithelialized hinge flap in our department. Because the direction of the scar was not following the relaxed tension line, the depression corrected successfully, but it left a linear scar. The thing she wanted more, was the correction of depression. Four months postoperatively, at a follow-up, she also achieved a good correction of the depressed scar (Fig. 4B). Patients were satisfied with treatment outcomes.

\section{DISCUSSION}

The skin is a complex organ. Once damaged due to trauma or infections, it is mainly healed through a simple process called fibrosis [6]. In this process, the accumulation of abnormal collagen causes the formation of various scars. The depressed scars of these would be formed unless the tissue defects are appropriately restored by the synthesis of collagen and connective tissue during the wound healing process. In the depressed scars, the tissue defects formed in the deep wounds are filled with the granulation tissue during the wound healing process. This leads to the fibrosis during the maturation process, followed by the tissue contraction leading to the increased skin tension. Thus, the depressed scars are formed [4]. After adhesiolysis in the deep skin layer, the fillers (e.g., collagen and hyaluronic acid), autologous fat and autologous dermofat grafting are used to correct the depressed scars [2]. Although the filler or autologus fat grafting are a simple procedure, they are disadvantageous in that the autologous fat grafting frequently causes complications such as necrosis and infections and it is extensively absorbed with an absorption rate of 50\% [7]. To overcome these disadvantages, Peer and Paddock used the dermofat grafting [8]. The dermofat grafting is characterized by the appropriate level of strength and rigidity. Moreover, it is less absorbed with a high rate of engraftment because the capillary vessels are distributed in the graft site [9-11]. But it is also problematic in that additional surgeries are needed for the donor site and this may lead to the formation of scars at the donor site. In addition, in cases of large-sized or deep depressed scar, there would be limitations in the correction of depressed scars.

To date, various methods have therefore been attempted to correct challenging cases of severe depressed scars [12,13]. In 1918, Poulard first described the methods for correcting depressed scars 
formed after tracheostomy [14]. Poulard performed de-epithelialization of the scar tissue and thereby left the scar tissue in the dermal layer. The residual scar tissue is helpful for the correction of depressed scar. This methods can be used very simply. However, due to an insufficient amount of the residual scar tissue, there is a limitation in the correction of the deep depressed scar. Then this method have been used for scar revision, in case that the depresion was not severe. Later on, in 1970, Lewin and Keunen modified the methods of Poulard, thus correcting the depressed scar after tracheostomy by making the shape of the de-epithelialized depressed scar tissue into a tube shape [4]. Thereafter, the hinge flap has been used to correct the post-tracheostomy scar. The hinge flap is also termed as 'turn-over or fold-over flap'. It was first described in 1980 when Leonard used the de-epithelialized deltopectoral flap to reconstruct the chest wall defects due to the radiation-induced necrosis of the chest wall [15]. This method is characterized by the correction of the depressed scar by turning over the dermis, subcutaneous and muscular layers like the page of the book [5]. It is such a good method that can correct the depressed scars by folding the tissue. However, it is also disadvantageous, in that new donor sites are needed and the skin graft is needed to cover the surface of the donor flaps (Fig. 1A). Scar revision alone can't correct the depressed scar, because the depression can't be corrected appropriately and the method of hinge flap is not also needed, because the extent of the depression is not large. Because of this case, we want to find proper new method.

We intended to correct the depressed scar effectively by using flaps, but attempted not to make new donor sites or additional skin grafts. As described earlier, new donor sites are not needed if the scar tissues are used as a flap like the methods of Poulard. It can therefore be inferred that it would be possible to correct the depressed scars without new donor flaps if surgeons fold the scar tissue like a hinge flap in the correction of deep scar tissue (Fig $1 B)$. We used our methods to correct the depressed scars after tracheostomy and depressed scars formed in the lower extremities, and the result was excellent. We have therefore speculated our methods would be effective in the correction of deep depressed scars occurring at various sites.

Currently, various methods are attempted to treat the depressed scars depending on their severity. But there are no established modalities in this series. The fillers are insufficient for correcting the long, deep depressed scars. They are disadvantageous in that they are absorbed over time and require new donor sites. In our new method, the flap which was composed of the scar tissues, was used to correct depressed scars. And we folded the scar tissue without harvesting the flaps from new donor sites which is different from hinge flap and thereby corrected the depressed scars. Thus, we obtained good treatment outcomes. Our methods are advantageous in that the procedure is simple and effective, but new donor sites or additional skin grafts are not needed. However, these techniques cause the problem that the scar becomes longer and amounts of scar tissue are insufficient for correcting the depression. To resolve the long scar, we place an incision within or parallel to a related skin tension line, if that's possible. Though the scar length in the neck, it is less visible. In case the direction of the scar was not following the relaxed tension line, it left a linear visible scar. But, the thing patient wanted more, was the correction of depression. After they achieved a good correction of the depressed scar, all of them were satisfied with treatment outcomes. And to increase the tissue requirements for correcting the depression, we overlaped the both ends of bilateral hinge flaps. In this method, a little more undermining of central area is necessary. However, the approximately middle $1 / 3$ of the center should remain, since it constitutes the vascular pedicle of the flap [4]. If the blood supply of this flap is compromised, it becomes absorption and shrinkage [4]. As the result, the scar will be depression gradually, but we don't experience it in our cases.

Briefly, we corrected the depressed scars by folding the de-epithelialized bilateral scar tissue. We assume that our methods would be simple and effective for the treatment of deep depressed scars occurring at various sites. Here, we report our methods and their outcomes with a review of literatures.

\section{REFERENCES}

1. Thomas JR, Prendiville S. Update in scar revision. Facial Plast Surg Clin North Am 2002;10:103-11.

2. Kontis TC. Scar Revision and skin resurfacing. In: Snow JB, Wackym PA, editors. Ballenger's Otorhinolaryngology Head and Neck Surgery. 17th ed. Connecticut: People's Medical Publishing House; 2009. p. 707-16.

3 Skigen AL, Bedrock RD, Stopperich PS. Correction of the depressed, retracted, post-tracheostomy scar. Plast Reconstr Surg. 1999;103:1703-5.

4. Lewin ML, Keunen HF. Revision of the post-tracheotomy scar: Correction of the depressed, retracted scar. Arch Otolaryngol. 1970;91:395.

5. Salmon PJ, Mortimer NJ, Hill SE. Muscular hinge flaps: Utility and technique in facial reconstructive surgery. Dermatol Surg 2010;36:227-34.

6. Peacock EE, Winkle WV. Wound Repair. Philadelphia: WB Saunders; 1976. p. 204-70.

7. Peer LA. Loss of weight and volume in human fat grafts. Plast Reconstr Surg 1950;5:217.

8. Peer LA, Paddock R. Histologic studies on the fate of deeply implanted dermal grafts. Arch Surg 1937;31:268.

9. Rees TD. The transfer of free composite grafts of skin and fat: Clinical study. Plast Reconstr Surg Transplant Bull 1960;25:556.

10. Smith RO Jr, Dickinson JT, Cipcic JA. Composite graft in facial reconstructive surgery. Arch Otolaryngol 1972;95:252. 
11. Hom DB, Winters M. Effects of angiogenic growth factors and a penetrance enhancer on composite grafts. Ann Otol Rhinol Laryngol 1998;107:769.

12. Grant N, Davison SP. Management of the post tracheostomy scar. Laryngoscope 2007;117:2107-9.

13. Johnson TM, Baker S, Brown MD, et al. Utility of the subcutane- ous hinge flap in nasal reconstruction. J Am Acad Dermatol 1994;30:459-66.

14. Poulard A. Traitement de cicatrices faciales. Presse Med 1918; 26:221.

15. Leonard AG. Reconstruction of the chest wall using a deepithelialised turn-over deltopectoral flap. Br J Plast Surg 1980;33:187-9. 